\title{
Méthodologie de caractérisation d'un faisceau laser de puissance
}

\author{
K. F. Badawi ( $\left.{ }^{1}\right)$, Th. Manderscheid $\left({ }^{2}\right)$ et P. Gorria $\left({ }^{1}\right)$ \\ (1) I.U.T. Le Creusot, France \\ ( $\left.{ }^{2}\right)$ P.S.A., Etudes et Recherche, Centre Technique Citroën, Vélizy, France
}

(Reçu le 11 octobre 1989, révisé et accepté le 7 décembre 1989)

\begin{abstract}
Résumé. - Une méthodologie pour caractériser un faisceau laser de puissance basée sur la mesure du diamètre du faisceau en fonction de la distance par rapport à sa taille, est présentée. Les différentes sources d'erreurs sont exposées ainsi que les précautions à prendre pour les éviter. L'application de la méthodologie à la caractérisation d'un faisceau laser YAG pulsé de $400 \mathrm{~W}$ de puissance est réalisée et discutée.
\end{abstract}

\begin{abstract}
A methodology for characterising a high power laser beam based on the measurement of the diameter of the beam as a function of the distance from the beam waist is described. The various sources of error are described as are the precautions to be taken to avoid them. The application of the beam characterisation methodology for a pulsed YAG laser beam of a power of $400 \mathrm{~W}$ is demonstrated and discussed.
\end{abstract}

\section{Introduction.}

Les lasers de forte puissance atteignant quelques dizaines de $\mathrm{kW}$ ou quelques gigawatt par $\mathrm{cm}^{2}$ de densité de puissance sont de plus en plus utilisés dans le traitement des matériaux (soudage, découpe, traitements et revêtements superficiels, marquage, etc.). La température de la surface traitée dépend essentiellement des caractéristiques du matériau et de celles du faisceau.

La connaissance précise de ces dernières caractéristiques est absolument indispensable à la maîtrise du traitement considéré. Nous présentons dans cet article une méthodologie de caractérisation d'un tel faisceau avec un exemple d'application sur un laser YAG de $400 \mathrm{~W}$ de puissance.

\section{Ra el e}

Un faisceau laser est régi par les équations de Maxwell et les conditions d'interférence dans la cavité résonnante. Pour un faisceau monomode, il est le produit d'une onde sphérique par une fonction exponentielle qui en atténue l'intensité en fonction de la distance à son axe. Les équations fondamentales de sa propagation à l'extérieur du résonateur sont données par: (voir Réfs. [1-4])

$$
\omega(Z)=\omega_{0}\left[1+\left(\frac{Z \lambda}{\pi \omega_{0}^{2}}\right)^{2}\right]^{1 / 2}
$$

$$
\text { et } \quad R(Z)=z\left[1+\left(\frac{\pi \omega_{0}^{2}}{Z \lambda}\right)^{2}\right] \text {. }
$$

Où $\lambda$ est la longueur d'onde du faisceau et les quantités $\omega(Z), \omega_{0}$ et $R(Z)$ sont indiquées sur la figure 1 .

De ces équations on tire les quantités suivantes :

a) La profondeur de champ égale à $2 Z_{\mathrm{r}}$, où $Z_{\mathrm{r}}$ est la distance de Rayleigh, donnée par l'équation :

$$
Z_{\mathrm{r}}=\frac{\pi \omega_{0}^{2}}{\lambda}
$$

Elle représente la distance où le rayon du faisceau est égale à $\omega_{0} \times \sqrt{2}$;

b) la divergence $\theta$ du faisceau définie par :

$$
\theta=\operatorname{limite}_{Z \rightarrow \infty} \frac{2 \omega(Z)}{Z}=\frac{2 \omega_{0}}{Z_{r}} .
$$

En remplaçant $Z_{\mathrm{r}}$ et $\theta$ par leurs valeurs, on obtient l'équation suivante :

$$
d^{2}=d_{0}^{2}+\theta^{2} Z^{2}
$$

qui est équivalente à l'équation (1), et où $d=2 \omega(Z)$, et $d_{0}=2 \omega_{0}=$ la taille du faisceau ; 


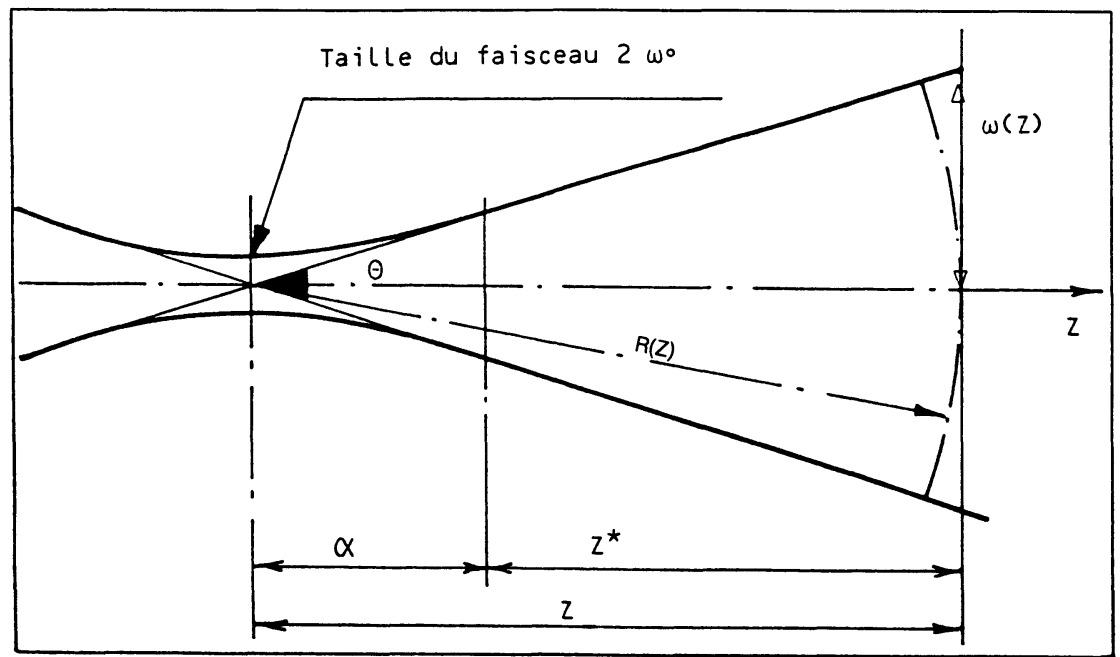

Fig. 1. - Représentation d'un faisceau laser.

[A representation of a laser beam.]

c) l'invariant de Lagrange Smith qui est défini par:

$$
I=d_{0} \theta=\frac{4 \lambda}{\pi} .
$$

C'est une constante caractéristique du faisceau en vertu des équations (3) et (4). Cet invariant joue un rôle très important dans l'utilisation des lasers de puissance puisqu'il permet de calculer avec précision les dimensions de la tache irradiée du matériau. En effet, dans la plupart des cas, le faisceau est focalisé au voisinage de la surface traitée pour atteindre des densités de puissance élevées. Dans la zone de focalisation, la mesure de la divergence $\theta^{\prime}$ est facile mais non celle de la taille du faisceau $d_{0}^{\prime}$, la connaissance de l'invariant de Lagrange permet alors d'atteindre $d_{0}^{\prime}$ par la relation :

$$
d_{0}^{\prime}=\frac{I}{\theta^{\prime}} .
$$

La surface irradiée est géométriquement calculable à partir de $d_{0}^{\prime}$, d'où la densité de puissance incidente au matériau.

Dans le cas d'un faisceau multimode (voir par exemple Ref. [3]) les équations fondamentales (1) et (2) restent valables en y remplaçant $\omega_{0}$ par $W_{0}=k \omega$ et $\omega$ par $W=k \omega$, où $k$ est une constante qui dépend des modes transverses $m, n$ par la relation:

$$
k=[(2 m+1)(2 n+1)]^{1 / 2} .
$$

Les autres caractéristiques du faisceau deviennent :

1) Divergence :

$$
\theta(\text { multimode })=k \theta(\text { monomode })=\frac{2 W_{0}}{Z_{\mathrm{r}}}
$$

où $Z_{\mathrm{r}}$ est la distance de Rayleigh du faisceau multimode.

2) Distance de Rayleigh :

$Z_{\mathrm{r}}($ multimode $)=\frac{\pi W_{0}^{2}}{k^{2} \lambda}=\frac{\pi \omega_{0}^{2}}{\lambda}=Z_{\mathrm{r}}$ monomode

La distance de Rayleigh ne change pas avec le mode.

3) L'invariant de Lagrange-Smith

$$
\begin{aligned}
I(\text { multimode }) & =d_{0}(\text { multimode }) \times \theta(\text { multimode }) \\
& =k d_{0}(\text { mono }) \times k \theta(\text { mono }) \\
& =k^{2} I(\text { monomode }) .
\end{aligned}
$$

L'équation (5) devient à son tour,

$$
D^{2}=D_{0}^{2}+\theta^{2} Z^{2}
$$

où $D$ est le diamètre du faisceau multimode au point $Z$, et $D_{0}$ est sa taille.

Les équations précédentes démontrent qu'il suffit de connaître $\theta$ et $D_{0}$ pour déduire $Z_{\mathrm{r}}, I$ et $k$. Il faut ajouter la connaissance de la répartition spatiotemporelle de son énergie et son état de polarisation pour compléter la caractérisation du faisceau.

Nous avons considéré dans d'autres études la répartition spatio-temporelle de l'énergie du faisceau (Réfs. [5-7]) et nous nous limiterons dans la présente aux 5 caractéristiques :

$$
\theta, D_{0}, Z_{\mathrm{r}}, I \text { et } k \text {. }
$$

\section{Méthodes expérimentales.}

3.1 Source LASER. - Nous avons utilisé une source YAG multimode pulsée de $400 \mathrm{~W}$ de puis- 
sance moyenne, polarisée aléatoirement et fabriquée par la Société LASER APPLICATION. Ses caractéristiques et ses performances ont été étudiées ailleurs (Réf. [8]). Nous avons effectué les mesures avec des pulses de 7,2 Joules, d'une durée de $1 \mathrm{~ms}$, à une fréquence de tir de $30 \mathrm{~Hz}$.

3.2 Mesure De la divergenCE ET DE LA TAILle DU FAISCEAU. - Nous avons développé pour cette mesure deux méthodes :

- La méthode de la double régression linéaire.

- La méthode de la régression polynomiale.

3.2.1 Méthode de la double régression linéaire. Cette méthode est composée de deux régressions linéaires. La première concerne les diamètres $D$ en fonction des $Z^{*}$ (voir Fig. 1) et sert à calculer la divergence $\theta$ et la position $\alpha$ de la taille du faisceau.

La deuxième concerne le carré du diamètre de la taille $D_{0}^{2}$ en fonction de $Z$, et sert à corriger $\alpha$ et à calculer $D_{0}$ avec précision.

En effet, à la limite de $Z \rightarrow \infty$, l'équation (11) devient :

$$
D=\theta \times Z
$$

Le faisceau se propage en ligne droite suivant les lois de l'optique géométrique. La divergence est la pente de la droite $D=f(Z)$. Généralement la position de la taille du faisceau n'est pas connue avec précision. L'origine des $Z$ est donc incertaine, et l'on est obligé de prendre une origine arbitraire située à une distance $\alpha$ inconnue de l'origine réelle (voir Fig. 1). Les $Z$ sont remplacés par des $Z^{*}$ égaux à $Z-\alpha$. L'équation (12) devient alors :

$$
D=\theta Z^{*}+\alpha \theta \text {. }
$$

Cette équation permet de calculer $\theta$ à partir des mesures de $D$ pour différents $Z^{*}$. Son ordonnée à l'origine permet de calculer $\alpha$, d'où la position de la taille du faisceau. Connaissant $\alpha$, on calcule les $Z$ à partir des $Z^{*}$ et l'équation (11) permet alors de calculer $D_{0}$ (la taille du faisceau) avec n'importe quel couple de points $Z, \theta$.

C'est la première régression linéaire.

En fait la valeur de $\alpha$ calculée à partir de (13) est souvent imprécise car l'ordonnée à l'origine d'une droite est d'autant plus imprécise que la droite est définie à partir de points expérimentaux loin de cette origine. Or l'équation (13) n'est valable que pour $Z \rightarrow \infty$, c'est-à-dire loin de l'origine. Par conséquent la précision sur $\alpha$ est forcément médiocre. Une erreur $\Delta \alpha$ sur cette grandeur entraîne une erreur $\Delta Z$ sur $Z$ égale à $\Delta \alpha$. L'équation (11) ne donne plus $D_{0}$ mais une valeur approchée $D_{0}^{*}$ de celui-ci. En effet l'équation :

$$
D_{0}^{2}=D^{2}-\theta^{2} Z^{2}
$$

devient $D_{0}^{* 2}=D^{2}-\theta^{2}(Z+\Delta \alpha)^{2}$

$$
=\left(D_{0}^{2}-\theta^{2} \Delta \alpha^{2}\right)-2 \theta^{2} \Delta \alpha Z
$$

or, $D_{0}, \theta$ et $\Delta \alpha$ sont des constantes, d'où

$$
D_{0}^{* 2}=A-\Delta \alpha B . Z
$$

où $A$ et $B$ sont des constantes.

C'est l'équation d'une droite en fonction de $Z$, dont la pente permet de calculer $\Delta \alpha$. C'est la deuxième régression linéaire de la méthode : une variation linéaire des $D_{0}^{2 *}$ en fonction de $Z$ démontre l'existence d'une erreur sur $\alpha$ et permet de calculer cette erreur.

Connaissant $\Delta \alpha$, on corrige $\alpha$ et les $Z$ et on recalcule $D_{0}$. Cette opération doit être recommencée jusqu'à l'annulation de $\Delta \alpha$.

3.2.2. Méthode de la régression polynomiale. - Une autre méthode consiste à ne pas se placer dans le champ lointain et à utiliser directement l'équation (11) dans laquelle on remplace $Z$ par $Z^{*}+\alpha$. On obtient alors :

$$
\begin{aligned}
& D^{2}=A+B Z^{*}+C Z^{* 2} \\
& \text { où } \quad A=D_{0}^{2}+\alpha^{2} \theta^{2} \\
& B=2 \alpha \theta^{2} \\
& \text { èt } \\
& C=\theta^{2} \text {. }
\end{aligned}
$$

En mesurant $D$ en fonction des $Z^{*}$, on calcule le polynôme de deuxième degré qui minimise l'écart quadratique moyen entre les points expérimentaux et la courbe du polynôme, et l'on détermine les coefficients $A, B$, et $C$ qui permettent de calculer $\theta$, $\alpha$ et $D_{0}$. Cette méthode est plus rapide que la précédente mais nous verrons plus loin qu'elle est moins précise.

\subsection{Mesure du diamètre du faisceau. - Nous} avons utilisé 2 méthodes courantes pour évaluer le diamètre d'un faisceau laser de forte puissance :

- la méthode du panier sensible;

- la méthode du diaphragme.

3.3.1 Méthode du papier sensible. - L'utilisation du papier sensible tel que le papier radio ou le papier $\mathrm{p}$ oto insolé permet de visualiser la forme géométrique du faisceau et le diamètre correspondant au seuil de brûlure du papier. Ceci limite le domaine d'utilisation de cette méthode à des densités de puissance supérieures à ce seuil.

3.3.2 Méthode du diaphragme. - Le diamètre du faisceau est mesuré à l'aide d'un système comportant 3 éléments (Fig. 2)

- un diaphragme à iris refroidi à l'air ;

- un joulemètre COHERENT modèle 213 ;

- un système de centrage à 3 axes. 


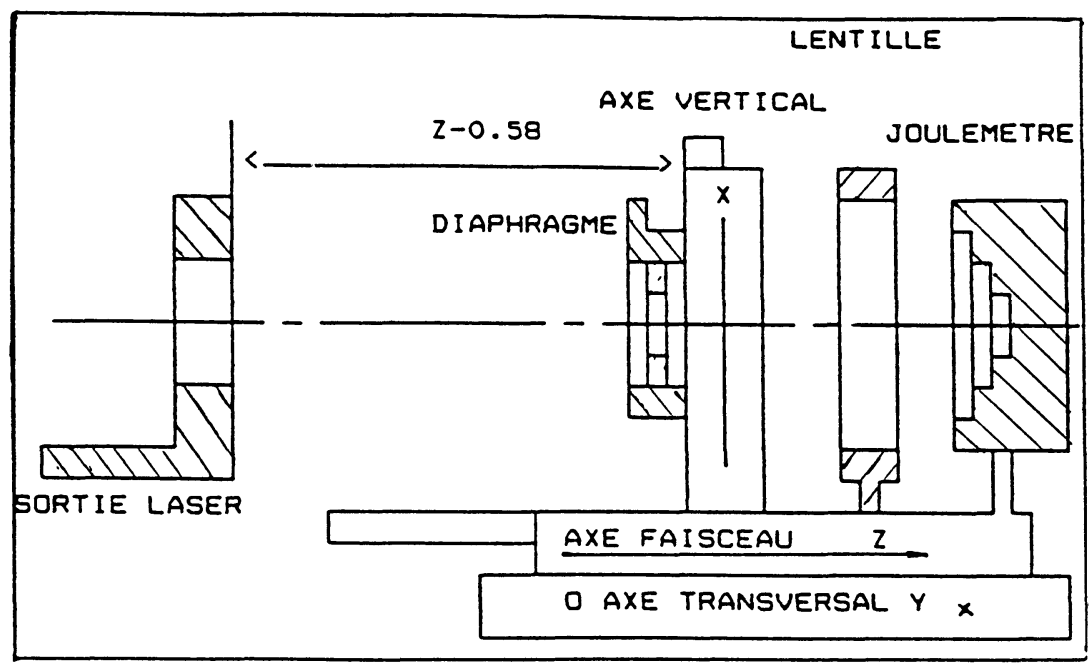

Fig. 2. - Système de mesure du diamètre du faisceau.

[System for measuring the diameter of the beam.]

Ce diamètre correspond à un passage de $86 \%$ de l'énergie totale du faisceau. La valeur choisie du seuil $(86 \%)$ résulte simplement d'une analogie avec les faisceaux monomodes gaussiens.

La distance de mesure est limitée à 4 mètres de la source. Au-delà de cette distance, la section du faisceau devenait elliptique et la mesure moins précise. Ce phénomène est en cours d'étude et sa discussion sera présentée dans un prochain article.

Nous avons évalué l'erreur sur la mesure du diamètre en faisant 2 mesures suivant 2 directions orthogonales avec le papier sensible et 3 mesures consécutives avec le diaphragme. Le tableau I montre les résultats obtenus. L'erreur commise sur le diamètre avec le diaphragme est de $\pm 0,1 \mathrm{~mm}$ correspondant à 2 fois l'écart type des diamètres mesurés, alors que celle du papier sensible est de $\pm 0,3 \mathrm{~mm}$.

Tableau I. - Diamètres mesurés avec le diaphragme. [Diameters measured using the diaphragm.]

\begin{tabular}{|c|l|l|l|}
\hline $\begin{array}{c}\text { Distance } \\
\mathrm{m} . \mathrm{m}\end{array}$ & $\begin{array}{c}D_{1} \\
\mathrm{~m} . \mathrm{m}\end{array}$ & $\begin{array}{c}D_{2} \\
\mathrm{~m} . \mathrm{m}\end{array}$ & $\begin{array}{c}D_{3} \\
\mathrm{~m} . \mathrm{m}\end{array}$ \\
\hline 200 & 4,7 & 4,85 & 4,9 \\
250 & 5 & 5 & 5 \\
300 & 5,15 & 5,20 & 5,25 \\
350 & 5,55 & 5,45 & 5,45 \\
400 & 5,65 & 5,5 & 5,55 \\
450 & 5,85 & 5,75 & 5,80 \\
500 & 6 & 6,05 & 5,85 \\
550 & 6,15 & 6,20 & 6,05 \\
600 & 6,35 & 6,30 & 6,35 \\
650 & 6,60 & 6,55 & 6,50 \\
800 & 6,75 & 6,7 & 6,75 \\
900 & 7 & 7,05 & 7,10 \\
\hline
\end{tabular}

Par ailleurs, le tableau II montre les diamètres mesurés par la méthode du papier sensible comparés à ceux obtenus par la méthode du diaphragme. Il montre que les premiers sont systématiquement plus grands que les seconds, et qu'il existe une variation importante du diamètre en fonction de la direction. Les coefficients de corrélation entre les 2 méthodes sont les suivants :

- Diaphragme - axe $X$ du papier : $\ell=0,994$.

- Diaphragme - axe $Y$ du papier : $\ell=0,99$.

Ils montrent que pour la suite de l'étude, on peut choisir l'une ou l'autre méthode. Nous avons choisi la première à cause de la clarté de définition du diamètre qu'elle donne.

Tableau II. - Comparaison des deux méthodes. [Comparaison of the two methods.]

\begin{tabular}{|c|c|c|c|}
\hline \multirow{2}{*}{ Distance } & mesures par & \multicolumn{2}{|c|}{ mesures papier radio } \\
\cline { 3 - 4 }$Z(\mathrm{~m})$ & $\begin{array}{c}\text { diaphragme } \\
d(\mathrm{~mm})\end{array}$ & $\begin{array}{c}\text { axe } X(1) \\
d(\mathrm{~mm})\end{array}$ & $\begin{array}{c}\text { axe } Y(2) \\
d(\mathrm{~mm})\end{array}$ \\
& & 11,0 & 10,0 \\
& & 11,8 & 10,5 \\
0,825 & 8,828 & 12,4 & 10,9 \\
0,972 & 9,684 & 12,6 & 11 \\
1,078 & 10,540 & 14 & 12 \\
1,176 & 10,540 & 16 & 14 \\
1,176 & 10,896 & 17,4 & 15,2 \\
1,570 & 12,323 & 13,821 & 15,8 \\
1,790 & 15,034 & 18,5 & 17 \\
1,966 & 16,674 & 19 & 18,5 \\
2,175 & 17,174 & 21 & 18,2 \\
2,398 & 18,600 & 22,6 & 20,4 \\
2,586 & 19,885 & 24,2 & \multicolumn{2}{|c}{} \\
\hline
\end{tabular}


3.4 EVAluATION DES ERREURS SUR LA DIVERGENCE.

3.4.1 Erreur fonctionnelle. - L'équation (12) est valable uniquement quand $Z \rightarrow \infty$. Les mesures du diamètre du faisceau doivent être faites loin de sa taille. L'erreur commise dans le cas du non-respect de cette condition est d'autant plus grande que l'on se rapproche de la taille. Mathématiquement, elle peut être estimée en calculant l'écart relatif entre le terme $\left[1+\left(\frac{Z}{Z_{\mathrm{r}}}\right)^{2}\right]^{1 / 2}$ correspondant à l'équation générale de propagation et le terme $\left(\frac{Z}{Z_{\mathrm{r}}}\right)$ qui le remplace à la limite des $Z \rightarrow \infty$. Nous appellerons cette erreur, l'erreur fonctionnelle $\lambda$. La figure 3 et le tableau III donnent les valeurs de $\lambda$ en fonction de $\frac{Z}{Z_{\mathrm{r}}}$ et les illustrent. Il y apparaît que pour $\frac{Z}{Z_{\mathrm{r}}}$ égal à 0,98 , l'erreur fonctionnelle est de $30 \%$. Elle se réduit à $10 \%$ pour $\frac{Z}{Z_{\mathrm{r}}}=2,06$. Par conséquent toute mesure de divergence doit respecter, au moins, la condition :

$$
\frac{Z}{Z_{\mathrm{r}}} \geqq 2
$$

qui limite l'erreur fonctionnelle à $10 \%$.

3.4.2 Erreur opératoire. - La mesure de la divergence nécessite la connaissance d'au moins 2 diamètres du faisceau à 2 endroits différents, comme le montre la figure 4.

$\theta$ est alors donnée par :

$$
\theta=\frac{\phi_{2}-\phi_{1}}{M} .
$$

Tableau III. - Erreur fonctionnelle en fonction de $Z / Z_{\mathrm{r}}$

[Variation in the functional error as a function of $Z / Z_{\mathrm{r}}$.]

\begin{tabular}{|c|c|}
\hline$\lambda$ & $Z / Z_{\mathrm{r}}$ \\
\hline $1 \%$ & 7,01 \\
$2 \%$ & 4,92 \\
$3 \%$ & 3,99 \\
$4 \%$ & 3,42 \\
$5 \%$ & 3,04 \\
$6 \%$ & 2,75 \\
$7 \%$ & 2,53 \\
$8 \%$ & 2,34 \\
$9 \%$ & 2,19 \\
$10 \%$ & 2,06 \\
$20 \%$ & 1,33 \\
$30 \%$ & 0,98 \\
$40 \%$ & 0,75 \\
$50 \%$ & 0,57 \\
$60 \%$ & 0,43 \\
$70 \%$ & 0,31 \\
$80 \%$ & 0,20 \\
$90 \%$ & 0,10 \\
\hline
\end{tabular}

L'erreur sur $\theta$ est donnée par

$$
\Delta \theta=\frac{2 \Delta \phi}{M}
$$

où $\Delta \phi$ est l'erreur sur la mesure des diamètres.

L'équation (17) montre que $\theta$ est d'autant plus précise que $\Delta \phi$ est petit et $M$ est grand. La figure 5 montre la variation de $\Delta \theta$ en fonction de $M$ et de

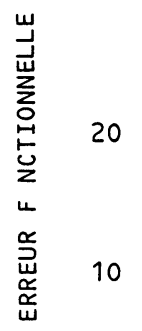

$\begin{array}{lllllll}1 & 2 & 3 & 4 & 5 & 6 & \mathrm{Z} / \mathrm{z}_{\mathrm{r}}\end{array}$

Fig. 3. - Variation de l'erreur fonctionnelle en fonction de $Z / Z_{\mathrm{r}}$.

[Variation of the functional error as a function of $Z / Z_{\mathrm{r}}$.] 


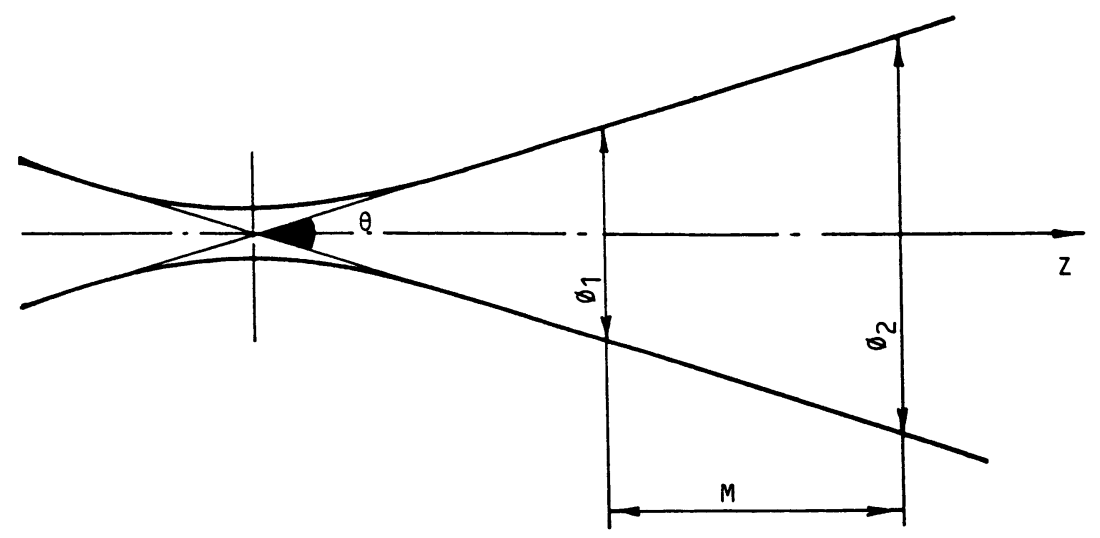

Fig. 4. - Mesure de la divergence.

[Divergence measurement.]

$\Delta \phi$. Pour que l'erreur opérationnelle soit inférieure à $0,5 \mathrm{mrad}$, il faut que l'intervalle de mesure soit supérieur à 2 mètres si l'erreur sur $\phi$ est inférieure à $0,5 \mathrm{~mm}$. Cette condition s'ajoute à la précédente et l'ensemble des figures 3 et 5 et du tableau III donne les moyens de choisir les conditions expérimentales optimales pour minimiser les erreurs sur la divergence.

3.4.3 Erreurs relatives à la méthode de la double régression linéaire. - Les deux paragraphes précédents ont montré qu'une mesure précise de la divergence exige l'utilisation d'un intervalle $M$ de $Z^{*}$ le plus grand possible et le plus éloigné possible de la taille du faisceau. Ce sont deux conditions difficiles à satisfaire simultanément, surtout quand on ne connaît pas a priori la distance de Rayleigh qui permet d'apprécier la position de l'intervalle de mesure par rapport à celle de la taille. D'où une erreur presqu'inévitable de la première mesure de $\theta$. Ceci entraîne souvent la nécessité de plusieurs itérations avant de déterminer sa valeur exacte.

D'autre part, nous avons vu qu'une régression linéaire des $D_{0}^{* 2}$ en fonction de $Z$ permet de calculer $\Delta \alpha$ directement. Ceci nous permet d'atteindre une valeur stable de $D_{0}$ après plusieurs itérations. Cette valeur est celle qui minimise l'écart type des $D_{0}$.

Mais nous avons remarqué que dans certains cas, le tableau des $D_{0}^{2}$ en fonction de $Z$ présentent quelques valeurs négatives. C'est la preuve d'une surestimation de $\alpha$, et on peut déduire d'office de sa valeur, la quantité qui annule les $D_{0}^{2}$ négatifs. Le tableau V (paragraphe 4) illustre un tel cas.

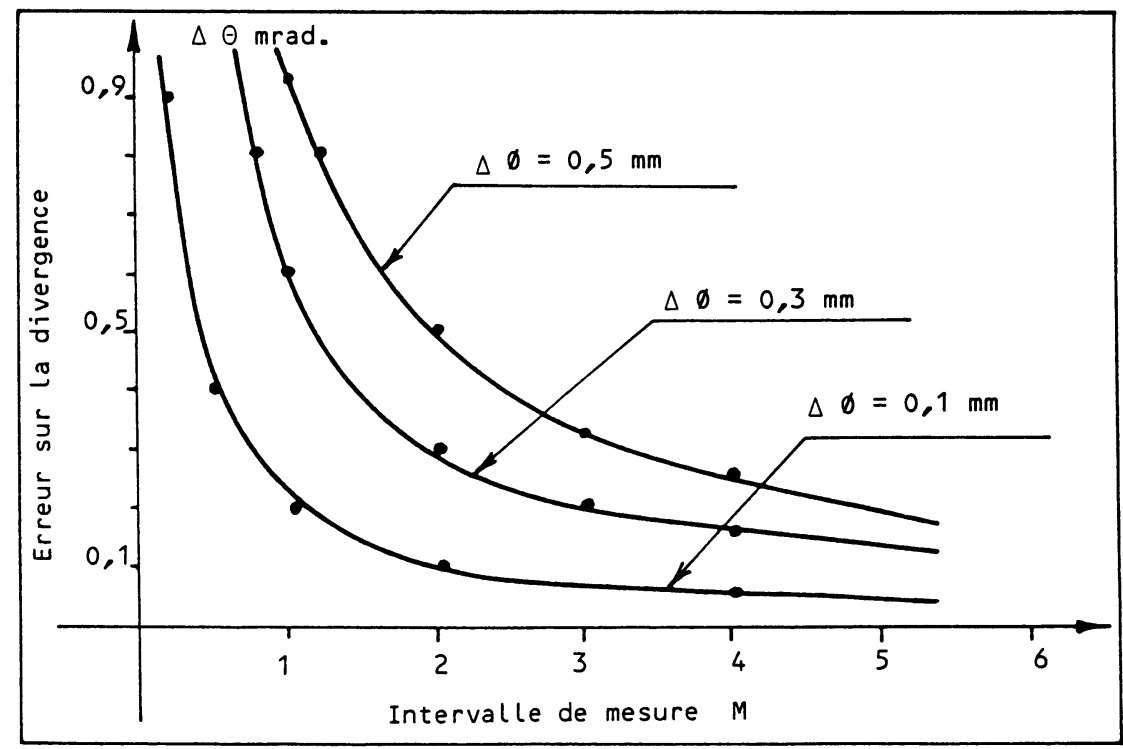

Fig. 5. - Erreur sur la divergence en fonction de $\Delta \phi$ et de $M$.

[Divergence error as a function of $\Delta \phi$ and $M$.] 
Après la stabilisation de $D_{0}$ et de $\alpha$, on procède au calcul des $Z_{\mathrm{r}}, I$ et $k$. Connaissant $Z_{\mathrm{r}}$, on refait le calcul de $\theta$ par l'équation (11), en corrigeant le choix du début de l'intervalle des $Z^{*}$. Ceci donne une nouvelle valeur de $\theta$. Connaissant cette nouvelle valeur, on recalcule les $D_{0}^{2}$ en fonction des $Z^{*}$ et on les stabilise comme décrit précédemment, et on recommence l'ensemble jusqu'à la stabilisation définitive de $\theta, \alpha$ et $D_{0}$. Le schéma suivant résume ce qui vient d'être dit :

1 : calcul de $\theta$ et $\alpha$ par la première régression linéaire ;

2: calcul des $D_{0}^{2}$ en fonction de $Z^{*}$;

3 : calcul des $\Delta \alpha$ par la $2^{\mathrm{e}}$ régression linéaire et retour à 2 jusqu'à la stabilisation des $D_{0}$;

4: nouveau calcul de $\theta$ et retour à 2 jusqu'à la stabilisation de $\theta$;

5 : calcul de $Z_{\mathrm{r}}, I$ et $k$.

3.4.4 Erreurs relatives à la méthode de régression polynomiale. - La régression polynomiale utilise une formulation unique pour décrire le faisceau en champ proche et lointain. Il s'ensuit la nécessité d'avoir des points expérimentaux du diamètre du faisceau dans ces deux champs. Sinon, les deux erreurs suivantes seront commises :

1 : Dans le cas où les points expérimentaux sont dans le champ lointain, la taille du faisceau et sa position seront erronées car elles ont des propriétés du champ proche obtenues par extrapolation du champ lointain.

2: Dans le cas contraire, la divergence sera erronée, comme l'ont montré les paragraphes 3.4.1 et 3.4.2.

Nous verrons des exemples de ces erreurs dans le paragraphe suivant.

\section{Applications.}

Nous avons appliqué les deux méthodes à l'exploitation des résultats présentés dans le tableau IV.

$\mathrm{La}$ régression polynomiale donne les résultats suivants :

$$
\begin{aligned}
\theta & =5,46 \mathrm{mrad} \\
\alpha & =1299 \mathrm{~mm} \\
0 & =-\quad, 7 D_{0} \text { imaginaire!) }
\end{aligned}
$$

Le diamètre imaginaire de la taille résulte du fait que les points expérimentaux sont dans le champ lointain et illustre les conclusions du paragraphe précédent.

La première itération de la méthode de double régression linéaire donne quant à elle les résultats suivants :

$$
\begin{aligned}
\theta & =6,24 \mathrm{mrad} \\
\alpha & =597 \mathrm{~mm}
\end{aligned}
$$

Tableau IV. - Diamètre du faisceau en fonction de $Z^{*}$.

[The diameter of the beam as a function of $Z^{*}$.]

\begin{tabular}{|c|c|}
\hline$*(\mathrm{~mm})$ & $D(\mathrm{~mm})$ \\
\hline 780 & 8,71 \\
825 & 8,83 \\
825 & 8,97 \\
880 & 9,26 \\
930 & 9,66 \\
972 & 9,68 \\
980 & 9,78 \\
1030 & 10,11 \\
1078 & 10,54 \\
1080 & 10,35 \\
1130 & 10,58 \\
1176 & 10,54 \\
1176 & 10,97 \\
1180 & 10,87 \\
1230 & 11,18 \\
1377 & 12,32 \\
1570 & 13,82 \\
1790 & 15,03 \\
1966 & 16,67 \\
2175 & 17,17 \\
2398 & 18,60 \\
2586 & 10,88 \\
2796 & 20,88 \\
\hline
\end{tabular}

$$
\begin{aligned}
D_{0} & =1,64 \mathrm{~mm} \\
I & =10,23 \mathrm{~mm} \cdot \mathrm{mrad} \\
Z_{\mathrm{r}} & =262 \mathrm{~mm} \\
k & =2,84 .
\end{aligned}
$$

Le début de l'intervalle de mesure se situe donc à $780+597=1377 \mathrm{~mm}$. Le rapport $Z / Z_{\mathrm{r}}$ correspondant est égal à 5,6. Par conséquent, l'erreur fonctionnelle sur $\theta$ est d'après le tableau III inférieure à $2 \%$. La longueur de l'intervalle étant de $2116 \mathrm{~mm}$ et $\Delta \phi$ de $0,1 \mathrm{~mm}$, l'erreur relative sur $\theta$ est ainsi

$$
\begin{aligned}
\frac{\Delta \theta}{\theta} & =\frac{\Delta \theta}{\theta} \text { opérationnelle }+\frac{\Delta \theta}{\theta} \text { fonctionnelle } \\
& -, \quad, \quad=\ldots, 0 .
\end{aligned}
$$

Le calcul des $D_{0}^{2}$ en fonction des $Z$ montre l'existence de multiples diamètres négatifs (Tab. V). La valeur de $\alpha$ obtenue par la première itération est donc surestimée. Ceci est confirmé par la dernière régression linéaire (celle des $D_{0}^{2}$ en fonction de $Z$ ) qui donne un $\alpha$ négatif et égal à $-19,3 \mathrm{~mm}$.

Après plusieurs itérations, on trouve la valeur définitive de $\alpha$ égale à $497 \pm 10 \mathrm{~mm}$ cette valeur est confirmée par le tableau VI qui donne les écarts types des $D_{0}$ en fonction de $\alpha$. On y remarque que 
Tableau V. - $D_{0}^{2}$ en fonction de $Z$ pour $\alpha=600 \mathrm{~mm}$.

[ $D_{0}^{2}$ as a function of $Z$ for $\alpha=600 \mathrm{~mm}$.]

\begin{tabular}{|c|c|c|}
\hline$Z(\mathrm{~mm})$ & $D_{0}^{2}\left(\mathrm{~mm}_{2}\right)$ & $D_{0}(\mathrm{~mm})$ \\
\hline 1380 & 2,69 & 1,64 \\
1425 & 1,12 & 1,06 \\
1480 & 1,49 & 1,22 \\
1530 & 3,23 & 1,79 \\
1572 & $-1,22$ & - \\
1580 & $-0,25$ & - \\
1630 & 0,12 & 0,34 \\
1678 & 2,86 & 1,69 \\
1680 & $-1,28$ & - \\
1730 & $-3,06$ & - \\
1776 & $-2,52$ & - \\
1780 & $-3,65$ & - \\
1830 & $-3,69$ & - \\
1977 & 1,58 & 2,26 \\
2170 & 9,98 & 3,16 \\
2390 & 6,45 & 2,53 \\
2566 & 24,90 & 4,99 \\
2775 & $-1,06$ & - \\
2998 & 0,46 & 0,67 \\
3186 & 5,15 & 2,27 \\
3396 & $-7,18$ & - \\
& & \\
\hline & & \\
\hline
\end{tabular}

c'est la valeur de 500 qui minimise effectivement l'écart type $\sigma\left(D_{0}\right)$ des $D_{0}$.

Le tableau VII donne par ailleurs, les valeurs des $D_{0}$ obtenues en fonction de $Z$. Nous remarquons que $D_{0}$ est très stable pour les faibles valeurs de $Z$, et qu'il varie fortement pour les valeurs élevées de $Z$. Ceci confirme le fait que les propriétés du champ proche doivent être évaluées à partir des points expérimentaux du champ proche. Par conséquent, nous prendrons pour $D_{0}$ la moyenne des valeurs correspondant aux points les plus proches de la taille. Le tableau VII montre que l'instabilité commence à partir du huitième point. Nous prendrons donc les 7 premiers points pour calculer $D_{0}$. Nous obtenons ainsi :

$$
D_{0}=3,5 \pm 0,17 \mathrm{~mm}
$$

Tableau VI. - Ecart type des $D_{0}$ en fonction de $\alpha$. [Standard deviation of $D_{0}$ as a function of $\alpha$.]
Tableau VII. $-D_{0}$ en fonction de $z$ pour $\alpha=500 \mathrm{~mm}$.

[ $D_{0}$ as a function of $Z$ for $\alpha=500 \mathrm{~mm}$.]

\begin{tabular}{|l|l|}
\hline$Z(\mathrm{~mm})$ & $D_{0}(\mathrm{~mm})$ \\
\hline 1280 & 3,596703 \\
1325 & 3,421399 \\
1380 & 3,533683 \\
1430 & 3,822703 \\
1472 & 3,235631 \\
1480 & $3,39236$. \\
1530 & 3,502622 \\
1578 & 3,920813 \\
1580 & 3,353161 \\
1630 & 3,138071 \\
\hline
\end{tabular}

Les résultats obtenus après stabilisation de $\alpha$ et de $D_{0}$ sont alors :

$$
\begin{aligned}
\theta & =6,24 \pm 0,31 \mathrm{mrad} \\
\alpha & =497 \mathrm{~mm} \\
D_{0} & =3,5 \pm 0,17 \mathrm{~mm} \\
Z_{\mathrm{r}} & =561 \mathrm{~mm} \\
I & =21,84 \mathrm{~mm} \cdot \mathrm{mrad} \\
k & =4,14
\end{aligned}
$$

Compte tenu de la nouvelle valeur de $Z_{\mathrm{r}}$ le calcul de $\theta$ doit être refait à partir de $Z$ égal à $1683 \mathrm{~mm}$ pour respecter la condition $Z / Z_{\mathrm{r}} \geqq 3$. Ceci donne $\theta=6,33 \pm 0,44 \mathrm{mrad}$.

Cette modification est négligeable et n'entraîne aucune conséquence significative sur les autres caractéristiques. Les résultats précédents peuvent donc être considérés comme définitifs.

\section{Conclusion.}

Dans cette étude, nous avons présenté une méthodologie pour caractériser un faisceau laser de puissance. Elle est basée sur la mesure du diamètre du faisceau en fonction de la distance par rapport à sa taille, et permet de déterminer les trois caractéristiques fondamentales du faisceau :

- la divergence $\theta$,

- la position de la taille,

- le diamètre de la taille $D_{0}$.

\begin{tabular}{|l|c|c|c|c|c|c|c|c|c|c|}
\hline$\alpha(\mathrm{mm})$ & 100 & 200 & 300 & 400 & 460 & 480 & 500 & 520 & 550 & 600 \\
\hline$\sigma\left(D_{0}\right)$ & 1,44 & 1,30 & 1,16 & 1,02 & 0,95 & 0,94 & 0,9 & 0,94 & 1 & 2 \\
\hline
\end{tabular}


(Il existe deux autres caractéristiques fondamentales que nous n'avons pas considérées dans cet article : la répartition spatio-temporelle de l'énergie du faisceau et sa polarisation).

Ces caractéristiques sont établies en utilisant deux méthodes différentes :

- la méthode de la régression polynomiale,

- la méthode de la double régression linéaire.

Les différentes sources d'erreur sont exposées et les précautions à prendre pour les éviter sont présentées. En particulier, il a été démontré que la mesure des diamètres doit couvrir les champs proche et lointain du faisceau, sinon, des erreurs importantes peuvent être commises, soit sur la divergence, soit sur la position et le diamètre de la taille.

A partir de ces trois caractéristiques, on déduit toutes les autres, à savoir :
- la distance de Rayleigh, $Z_{\mathrm{r}}$ égal au rapport $D_{0} / \theta$,

- la constante de Lagrange - Smith, $I=D_{0} \times \theta$.

Le facteur de mode donné par :

$$
k=\left[\frac{\pi \cdot D_{0} \theta}{4 \lambda}\right]^{1 / 2} \text {. }
$$

Nous avons appliqué cette méthodologie à la caractérisation d'un faisceau laser YAG pulsé de $400 \mathrm{~W}$ de puissance et montré la nécessité de plusieurs itérations avant d'établir les valeurs correctes de ses caractéristiques.

Nous pensons que grâce à l'utilisation de plus en plus répandue des lasers de puissance dans l'industrie, la méthodologie présentée ici peut rendre des services très utiles.

\section{Bibliographie}

[1] Maillet H., Le laser, principes et techniques d'application, coordinateur (Editions Lavoisier) Paris (1986)

[2] VAnnes A. B., Lasers et industries de transformation, coordinateur (Editions Lavoisier) Paris (1986).

[3] O'SHEA D. C., Elements of modern optical design (Wiley, Interscience editor, New York) 1985.

[4] KogelniK H., Li T., Laser Beam and Resonators, Proc I.E.E.E. 54 (1966) 1312, 1329.

[5] Badawi K., Jacrot G., ANDrzejewski H., KAHLOUN C., Influence de la forme du pulse d'un laser YAG sur le soudage de l'acier Z 2 CN 1712 et de l'AG. 4, 4c colloque interna- tional sur le soudage et la fusion par faisceau d'électrons et laser, Cannes (Sept. 1988).

[6] Badawi K., Paindavoine M., Jacrot G., Effets de la distribution spatio-temporelle de l'énergie du pulse sur le soudage par faisceau laser YAG, Sci. Rev. Métallurgie (Mars 1989) pp. 161-170.

[7] Badawi K., Paindavoine M., Jacrot G., The effects of the space time distribution of the pulse energy on YAG beam welding, Laser 5, Ed. S. K. Ghosh (April 1989) pp. 102-107.

[8] Badawi K., Jacrot G., ANDRZEJewski H., KAHLOUN.C., Etude paramétrique de l'usinage au laser YAG pulsé de $450 \mathrm{~W}$, Revue Souder 12 (1988) 139-146. 